\title{
NOTE ON EPIMORPHISMS AND MONOMORPHISMS IN HOMOTOPY THEORY
}

\author{
PETER HILTON AND JOSEPH ROITBERG
}

\begin{abstract}
We study epimorphisms $e: X \rightarrow X$ and monomorphisms $m: X \rightarrow X$ in the pointed homotopy category of path-connected CW-spaces. Our principal theorems allow us to infer that under suitable finiteness and fundamental group assumptions, such are in fact homotopy equivalences.
\end{abstract}

1. Recall that a group $G$ is said to be Hopfian if every epimorphism $\varepsilon: G \rightarrow G$ is an automorphism. The concept was introduced by Heinz Hopf $[\mathbf{H}]$ in his study of the classification of surface mappings and has proved of interest in group theory; for example, Graham Higman presented the first example of a finitely presented non-Hopfian group. Recently certain properties of pseudo-identities of locally nilpotent groups were observed [HR] to be related to the basic yet elementary fact that finitely generated nilpotent groups are Hopfian.

Now there is a theory of pseudo-identities in the (pointed) homotopy category $\mathcal{H}$ of path-connected CW-spaces [CHR] and this motivates our study here of Hopfian objects of $\mathcal{H}$; this notion plainly makes sense in any category since epimorphisms are categorically defined. It will turn out that nilpotent spaces of finite type are, in fact, Hopfian objects of $\mathcal{K}$.

It is natural to consider the dual notion of co-Hopfian object, based on the study of self-monomorphisms. In the category $\mathcal{G}$ of groups, the most obvious co-Hopfian objects are the finite groups (these are, of course, also Hopfian), but there are other examples. For example, any nilpotent group whose $p$-torsion subgroup is finite for each $p$ and such that the quotient by the torsion subgroup is a finite direct product of copies of the rationals is co-Hopfian (again, such groups are also Hopfian). It turns out that we can characterize self-monomorphisms in $\mathcal{H}$, provided that we confine attention to spaces whose higher homotopy groups are finitely generated. We deduce that if such spaces have co-Hopfian fundamental groups they are co-Hopfian objects of $\mathcal{H}$.

The adjunction equivalence

$$
[X, K(G, 1)] \cong \operatorname{Hom}\left(\pi_{1} X, G\right)
$$

Received by the editors January 24, 1983 and, in revised form, May 12, 1983.

1980 Mathematics Subject Classification. Primary 55D10, 55D30.

Key words and phrases. Epimorphism, monomorphism, Hopfian object, co-Hopfian object.

(C)1984 American Mathematical Society $0002-9939 / 84 \$ 1.00+\$ .25$ per page 
where [, ] denotes the set of (pointed) homotopy classes of maps, leads to the immediate inference

Proposition 1. If $e: X \rightarrow Y$ is an epimorphism in $\mathcal{H}$, then $e_{*}: \pi_{1} X \rightarrow \pi_{1} Y$ is an epimorphism in $\mathcal{G}$.

Corollary 2. If $G$ is a Hopfian group, then $K(G, 1)$ is a Hopfian object of $\mathcal{H}$.

The converse of this assertion may well fail. It is easy to construct a map $f$ : $K\left(G_{1}, 1\right) \rightarrow K\left(G_{2}, 1\right)$ which is not an epimorphism although $f_{*}: G_{1} \rightarrow G_{2}$. Thus, if $G_{2}$ is a group having a non-0 cohomology group $H^{i}\left(G_{2} ; \Gamma\right)$ (constant coefficients) $i \geqslant 2$, and if $G_{1}$ is a free group mapping onto $G_{2}$ (e.g., $\mathbf{Z} \rightarrow \mathbf{Z} / 2$ ), then $f$ is not an epimorphism in $\mathcal{H}$. From this example we readily construct a group $G$, namely $\left(\oplus_{i=-\infty}^{0} G_{1}\right) \oplus\left(\oplus_{j=1}^{\infty} G_{2}\right)$, and a "shift" map $f: K(G, 1) \rightarrow K(G, 1)$ which is not an epimorphism but such that $f_{*}: G \rightarrow G$. The existence of such maps $f$ is certainly an obstacle to any simple-minded proof of a converse to Corollary 2.

Dual to Proposition 1 is

Proposition 1*. If $\mu: G \rightarrow H$ is a monomorphism in $\mathcal{G}$ then $K(\mu, 1): K(G, 1) \rightarrow$ $K(H, 1)$ is a monomorphism in $\mathcal{H}$.

Corollary $2^{*}$. If $K(G, 1)$ is a co-Hopfian object of $\mathcal{H}$, then $\mathcal{G}$ is a co-Hopfian group.

However, the converse of Corollary $2^{*}$ is true since the converse of Proposition $1^{*}$ is true.

2. Epimorphisms in $\mathcal{H}$. We say that $X \in \mathcal{H}$ is of homologically finite type (hft) if $H_{i} X$ is finitely generated for $i \geqslant 1$.

THEOREM 3. If $X$ is hft and $e: X \rightarrow X$ is an epimorphism in $\mathcal{H}$, then $e_{*}: H_{i} X \cong H_{i} X$, $i \geqslant 0$.

Proof. For any (constant) coefficient group $G$, we have $e^{*}: H^{i}(X ; G)>H^{i}(X ; G)$, $i \geqslant 0$. If we take $G=\mathbf{Z} / p$ and pass to homology, we infer that $e_{*}$ : $H_{i}(X ; \mathbf{Z} / p) \rightarrow H_{i}(X ; \mathbf{Z} / p), i \geqslant 0$. But $H_{i}(X ; \mathbf{Z} / p)$ is finite since $X$ is hft, so that $e_{*}: H_{i}(X ; \mathbf{Z} / p) \cong H_{i}(X ; \mathbf{Z} / p), i \geqslant 0$. We now appeal to [HMR, Theorem II.1.14], again exploiting the fact that $X$ is hft, to infer that $e_{*}: H_{i} X \cong H_{i} X, i \geqslant 0$.

Corollary 4. If, in addition, $X$ is nilpotent, then $e: X \rightarrow X$ is a homotopy equivalence.

Proof. We know that a homology equivalence of nilpotent spaces is a homotopy equivalence.

Theorem 3 admits the following generalization.

Theorem 5. Let $f: X \rightarrow Y, g: Y \rightarrow X$ be epimorphisms in $\mathcal{H}$ and let $X$ be hft. Then $Y$ is $h f t$ and $f$ and $g$ are homology equivalences. 
Proof. By Theorem 3 we know that $g_{*} f_{*}: H_{i} X \cong H_{i} X, i \geqslant 0$. Thus $f^{*} g^{*}$ : $H^{i}(X ; G) \cong H^{i}(X ; G)$ for any (constant) coefficient group $G$. But $f^{*}: H^{i}(Y ; G)>$ $H^{i}(X ; G)$, so that $f^{*}: H^{i}(Y ; G) \cong H^{i}(X ; G)$; and, of course, $g^{*}: H^{i}(X ; G) \cong$ $H^{i}(Y ; G)$. Let $Z$ be the mapping cone of $f$. Then $H^{i}(Z ; G)=0, i \geqslant 1$, for any (constant) coefficient group $G$. From this it is easy to deduce that $H_{i} Z=0, i \geqslant 1$, so that $f_{*}: H_{i} X \cong H_{i} Y, i \geqslant 0$. Similarly, $g_{*}: H_{i} Y \cong H_{i} X, i \geqslant 0$.

Corollary 6. If, in addition, $X$ and $Y$ are nilpotent, then $f$ and $g$ are homotopy equivalences.

3. Monomorphisms in $\mathcal{H}$. Let $p: \tilde{X} \rightarrow X$ be a covering map. Then certainly $p$ is a monomorphism in $\mathfrak{H}$. In particular, if there is a map $m: X \rightarrow X$ inducing a monomorphism $\mu: \pi>\pi$ where $\pi=\pi_{1} X$ and if $p: \tilde{X} \rightarrow X$ is the covering map with $\pi_{1} \tilde{X}=\mu \pi$, then $m$ factors as $p h$, where $h: X \rightarrow \tilde{X}$. Moreover, $h$ is a homotopy equivalence provided that $m$ induces an automorphism of higher homotopy groups; and, in that case, $m$ is a monomorphism.

We now prove a converse of this, provided we impose a restriction on $X$. We say that $X \in \mathfrak{H}$ is of homotopically finite type (Hft) if $\pi_{i} X$ is finitely generated for $i \geqslant 2$.

TheOREM 7. If $X$ is $H f t$ and $m: X \rightarrow X$ is a monomorphism in $\mathcal{H}$, then $m=p h$, where $h$ is a homotopy equivalence $h: X \rightarrow \tilde{X}$ and $p: \tilde{X} \rightarrow X$ is a covering map with $\pi_{1} \tilde{X}=m_{*} \pi_{1} X$.

Proof. We invoke the theory of homotopy groups with coefficients. For $n \geqslant 3$, we define $^{1}$

$$
\pi_{n}(X ; G)=[M(G, n), X],
$$

where $M(G, k)$ is the co-Moore space, being a l-connected space with single nonvanishing integral cohomology group $G$ in dimension $k$.

In fact, we will only need (3.1) with $G=\mathbf{Z} / p$. It is then even possible to define the pointed set

$$
\pi_{2}(X ; \mathbf{Z} / p)=\left[S^{1} \cup_{p} e^{2}, X\right] .
$$

Recall that the cofibration sequence

$$
S^{1} \stackrel{p}{\rightarrow} S^{1} \rightarrow S^{1} \cup_{p} e^{2}
$$

gives rise to a long exact sequence

$$
\begin{aligned}
\cdots \rightarrow \pi_{n} X \stackrel{p}{\rightarrow} \pi_{n} X \rightarrow \pi_{n}(X ; \mathbf{Z} / p) \rightarrow \pi_{n-1} X & \stackrel{p}{\rightarrow} \pi_{n-1} X \rightarrow \cdots \\
& \rightarrow \pi_{2}(X ; \mathbf{Z} / p) \rightarrow \pi_{1} X \stackrel{p}{\rightarrow} \pi_{1} X
\end{aligned}
$$

\footnotetext{
${ }^{1}$ We depart here from the Eckmann-Hilton definition; we will only be concerned with coefficient groups $G$ which are finitely generated.
} 
and hence to a universal coefficient sequence

$$
\pi_{n} X \otimes \mathbf{Z} / p>\pi_{n}(X ; \mathbf{Z} / p) \rightarrow \operatorname{Tor}\left(\pi_{n-1} X ; \mathbf{Z} / p\right), \quad n \geqslant 3 .
$$

For $n=2$, we still have the embedding

$$
\pi_{2} X \otimes \mathbf{Z} / p>\pi_{2}(X ; \mathbf{Z} / p) \text {. }
$$

We propose to show that $m_{*}: \pi_{n} X \cong \pi_{n} X, n \geqslant 2$. We mimic the proof of [HMR, Theorem [I.1.14] and show that $m_{*_{1}}: \pi_{n} X \otimes \mathbf{Z} / p \cong \pi_{n} X \otimes \mathbf{Z} / p$ and $m_{*_{2}}$ : $\operatorname{Tor}\left(\pi_{n} X, \mathbf{Z} / p\right) \cong \operatorname{Tor}\left(\pi_{n} X, \mathbf{Z} / p\right)$ for all $p$ and $n \geqslant 2$, whence, since $X$ is $\mathrm{Hft}$, the conclusion follows. We first show that $m_{*_{1}}$ is an automorphism. Now since $m$ is a monomorphism, we have $m_{*}: \pi_{n}(X ; \mathbf{Z} / p)>\pi_{n}(X ; \mathbf{Z} / p), n \geqslant 2$. Thus, by (3.3) or (3.4), $M_{* 1}$ is a monomorphism. But, since $X$ is $\mathrm{Hft}, \pi_{n} X \otimes \mathbf{Z} / p$ is a finite group, so $m_{*_{1}}$ is an automorphism. We now prove that $m_{*_{2}}$ is an automorphism. For we have, with $n \geqslant 3$,

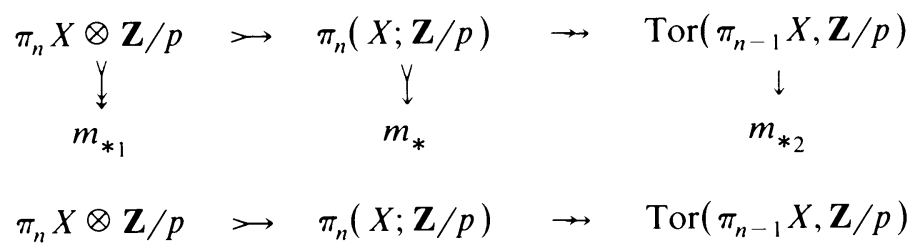

from which it follows that $m_{*_{2}}$ is a monomorphism. But again since $X$ is $\mathrm{Hft}, m_{*_{2}}$ is an automorphism. We have therefore established that $m_{*}: \pi_{n} X \cong \pi_{n} X, n \geqslant 2$, and, of course, $m_{*}: \pi_{1} X \gg \pi_{1} X$. The conclusion of the theorem therefore follows. ${ }^{2}$

COROllary 8 . If, in addition, $\pi_{1} X$ is co-Hopfian, then $m$ is a homotopy equivalence.

We generalize just as in the previous section.

TheOREM 9. Let $f: X \rightarrow Y, g: Y \rightarrow X$ be monomorphisms in $\mathcal{H}$ and let $X$ be Hft. Then $Y$ is Hft and factors as $X \stackrel{h}{\rightarrow} \tilde{Y} \stackrel{p}{\rightarrow} Y$, where $h$ is a homotopy equivalence and $p$ is a covering map.

Proof. We know that $g_{*} f_{*}: \pi_{n} X \cong \pi_{n} X, n \geqslant 2$ and $g_{*}: \pi_{n} Y>\pi_{n} X, n \geqslant 1, f_{*}$ : $\pi_{n} X \supset \pi_{n} Y, n \geqslant 1$. Thus $g_{*}: \pi_{n} Y \cong \pi_{n} X, n \geqslant 2$, and $f_{*}: \pi_{n} X \cong \pi_{n} Y, n \geqslant 2$. We now lift $f$ into $\tilde{Y}$, where $\pi_{1} \tilde{Y}=f_{*} \pi_{1} X$. The lifted map $h$ must be a homotopy equivalence. Of course there is a similar factorization of $g$.

COROllary 10. If, in addition, $\pi_{1} X$ or $\pi_{1} Y$ is co-Hopfian, then $f$ and $g$ are homotopy equivalences.

We remark finally that the results of $\S \S 2$ and 3 admit evident $P$-versions, where $P$ is a family of primes, provided that we confine attention to nilpotent spaces (compare the Appendix of $[\mathbf{H R}]$ ).

\footnotetext{
${ }^{2}$ The proof just given does not use the full force of the assumption that $m$ is a monomorphism. Thus, if we were to use the fact that $m_{*}: \pi_{n} X \succ \pi_{n} X, n \geqslant 2$, then it would suffice simply to establish that $m_{*_{1}}$ : $\pi_{n} X \otimes \mathbf{Z} / p \cong \pi_{n} X \otimes \mathbf{Z} / p$ for all $p, n \geqslant 2$.
} 


\section{BIBLIOGRAPHY}

[CHR] M. Castellet, P. Hilton and J. Roitberg, On pseudo-identities. II (in preparation).

[HMR] P. Hilton, G. Mislin and J. Roitberg, Localization of nilpotent groups and spaces, North-Holland Math. Studies, Vol. 15, Amsterdam, 1975.

[HR] P. Hilton and J. Roitberg, On pseudo-identities. I, Arch. Math. (to appear).

[H] H. Hopf, Beiträge zur Klassifizierung der Flächenabbildungen, J. Reine Angew. Math. 165 (1931). 225-236.

Department of Mathematics, SUNy at Binghamton, Binghamton, New York 13901

Graduate Center, City University of New York, 33 W. 42nd Street, New York, New York 10036

Hunter College, 695 Park Avenue, New York, New York 10021 